\title{
An ordinary differential equation model for the multistep transformation to cancer
}

\author{
Sabrina L. Spencer ${ }^{a, b, *}$, Matthew J. Berryman ${ }^{b}$, \\ José A. García ${ }^{c}$, and Derek Abbott ${ }^{b}$
}

\begin{abstract}
${ }^{a}$ Department of Human Genetics, University of Michigan, Ann Arbor, MI 48109-0618, USA. ${ }^{b}$ Center for Biomedical Engineering and School of Electrical \& Electronic Engineering, The University of Adelaide, SA 5005, Australia. ${ }^{c}$ Laboratory of Theoretical Biology, Universidad La Salle, 06140 México, D.F., México.

* Corresponding author. Email: sabrinal@umich.edu, Telephone: +1-856-327-5283
\end{abstract}

\begin{abstract}
Cancer is viewed as a multistep process whereby a normal cell is transformed into a cancer cell through the acquisition of mutations. We reduce the complexities of cancer progression to a simple set of underlying rules that govern the transformation of normal cells to malignant cells. In doing so, we derive an ordinary differential equation model that explores how the balance of angiogenesis, cell death rates, genetic instability, and replication rates give rise to different kinetics in the development of cancer. The key predictions of the model are that cancer develops fastest through a particular ordering of mutations and that mutations in genes that maintain genomic integrity would be the most deleterious type of mutations to inherit. In addition, we perform a sensitivity analysis on the parameters included in the model to determine the probable contribution of each. This paper presents a novel approach to viewing the genetic basis of cancer from a systems biology perspective and provides the groundwork for other models that can be directly tied to clinical and molecular data.

Keywords: cancer; oncogenesis; genetic instability; multistep transformation; ordinary differential equation model
\end{abstract}

\section{Introduction}

The standard perspective on cancer progression is that it is a form of somatic evolution where certain mutations give one cell a selective growth advantage (Cahill et al., 1999). Oncogenesis is thought to require several independent, rare mutation events to occur in the lineage of one cell (Nowell, 1976). Kinetic analyses have shown that four to six rate-limiting stochastic mutational events are required for the formation of a tumor Armitage and Doll, 1954: Renan, 1993). Hanahan and Weinbero (2000) proposed the following six hallmark capabilities that a normal cell must acquire to become a cancer cell: (i) self-sufficiency in growth signals, (ii) insensitivity to anti-growth 
signals, (iii) evasion of apoptosis, (iv) limitless replicative potential, (v) sustained angiogenesis, and (vi) tissue invasion and metastasis. They define genetic instability as an "enabling characteristic" that facilitates the acquisition of other mutations due to defects in DNA repair processes. We reduce these characteristics to the following four: angiogenesis $(A)$, immortality, including evasion of cell death $(D)$, genetic instability, a function of mutation rates $(G)$, and increased replication rate $(R)$. We consider invasion and metastasis $(M)$ as a final step that allows the spread of a localized tumor. In line with the views of Hanahan and Weinberg (2000), we foresee cancer research developing into a logical science where the molecular and clinical complexities of the disease will be understood in terms of a few underlying principles. We therefore explore the multistep progression to cancer with an ordinary differential equation (ODE) model which, despite the apparent complexity of the equations, is based on basic principles and a minimal set of parameters.

\section{Structure and parameters of the model}

Although the model applies to the process of oncogenesis in general, the parameters are loosely based on breast cancer data. We consider the following cell populations: a population of $10^{8}$ normal cells $(N)$, cells which have acquired the ability to induce angiogenesis $(A)$, cells with mutations which allow them to avoid death $(D)$, cells with mutations that lead to genetic instability $(G)$, cells with mutations which increase their replication rate $(R)$, and cells with two or more of these mutations. Cell populations that have acquired two or three mutations are denoted by listing the mutations together in alphabetical order (state $D R A$ would be listed as state $A D R$, for example). We label a cell which has acquired all four mutations a primary tumor cell $(T)$. Although our model only addresses development of a primary tumor in genetic detail, we allow a primary tumor cell that has acquired the capability to invade and metastasize to become a metastatic cell $(M)$.

The spontaneous mutation rate in human cells has been estimated to be in the range of $10^{-7}$ to $10^{-6} \mathrm{mu}-$ tations/gene/cell division (Jackson and Loeb, 1998). We assume a spontaneous mutation rate of $k_{1}=10^{-7}$ mutations/gene/cell division. The loss of DNA repair genes can increase the mutation rate by a factor ranging from $10^{1}$ to $10^{4}$ (Tomlinson et al., 1996). We assume that the mutation rate after a genetic instability mutation increases 1000-fold to $k_{2}=10^{-4}$ mutations/gene/cell division. Successful invasion and metastasis depend upon acquisition of the other hallmark capabilities, as well as several new capabilities (Hanahan and Weinberg, 2000). To simplify the model, we do not address the multistep progression of a tumor cell to a metastatic cell, and instead consider this complex process as one step. It has been estimated that the rate of successful metastasis is in the range of $10^{-9}$ to $10^{-7}$ per cell division (Luebeck and Moolgavkar, 2002), and so we use a conservative estimate of $k_{3}=10^{-9}$ for the transition from a primary tumor cell to a metastatic cell.

A tumor cannot grow past about $10^{6}$ cells without angiogenesis supplying blood to the tumor (Folkman, 1990). We thus cap the size of the tumor at $10^{6}$ cells until at least $10 \%$ of the population of non-normal, non-metastatic cells has acquired a mutation in an $A$ gene. This accounts for the fact that only a fraction of the cells in a tumor need to send angiogenesis signals in order to develop an adequate blood supply for the tumor. In addition, populations of non-normal, non-metastatic cells are always capped by a lethal tumor burden limit of $10^{13}$ cells (Friberg and Mattson, 1997), irrespective of the angiogenesis cap.

A survey of microarray data published on number of genes up- or down-regulated in various cancers Amatschek et al., 2004; Ernst et al., 2002; Nakamura et al., 2004; Zou et al., 2002) led us to estimate that there are approximately 400 genes involved in development of a primary tumor. Dividing by four categories, we assume that there are approximately 100 genes involved in each of categories $A, D, G$, and $R$. To account for the fact that some genes function in more than one category, we allow double and triple state transitions but reduce the number of genes involved to the order of 10 and 1, respectively, to reflect the likelihood that a single mutation would affect more than one category. For example, we assume there are 100 genes involved transitions where only one mutation is acquired (e.g. $N \rightarrow A, D \rightarrow D R, A G \rightarrow A G R$, or $A D G \rightarrow A D G R$ ), 10 genes involved in transitions where two mutations are acquired in one step (e.g. $N \rightarrow A D, G \rightarrow A D G$, or $A R \rightarrow A D G R$ ), and 1 gene involved in transitions where three mutations are acquired in one step (e.g. $N \rightarrow A D G$ or $G \rightarrow A D G R$ ). This feature accounts for a mutational hit in p53, for example, which could take a cell directly from $\mathrm{N}$ to DGR, as p53 is 
involved in apoptosis, DNA repair, and cell cycle progression (Vogelstein et al., 2000).

We estimate that the relative contribution to increased net proliferation for mutations in the $D$ and $R$ categories is 0.7 and 0.3, respectively, an inference made from work by Tomlinson and Bodmer (1995). Using this $D: R$ ratio of 0.7:0.3, a tumor volume doubling time for breast cancer of 500 days (Friberg and Mattson, 1997), and a cell division rate for breast cancer of $1 / 10.00$ days $^{-1}$ (Rew and Wilson, 2000), we calculate (see section 4) the following: cells without a mutation in an $R$ gene divide every $b=10.00$ days, cells with a mutation in an $R$ gene divide every $b_{R}=9.89$ days, the lifetime of cells without a mutation in a $D$ gene is $d=10.00$ days, and the lifetime of cells with a mutation in a $D$ gene is $d_{D}=10.16$ days. The birth and death rates are equal for normal cells and for all cells without a mutation in $D$ or $R$.

The above information is depicted in a unified fashion in Figure 1 and the parameters appearing in the ODE model are given in Table 1

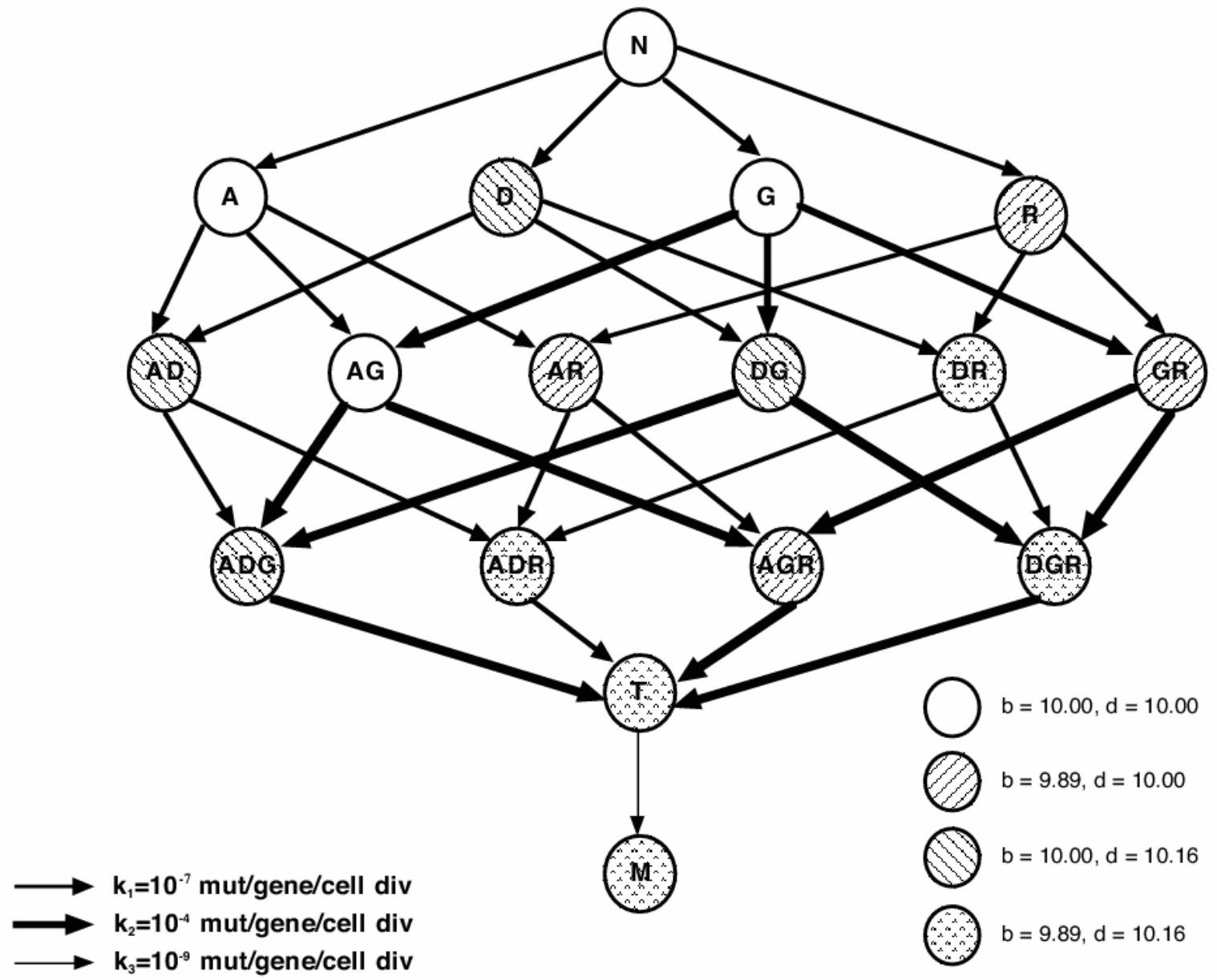

Figure 1. State diagram of the model. Normal cells $(N)$ can acquire mutations which give the cell the capability to induce angiogenesis $(A)$, mutations which give the cell the capability to avoid death $(D)$, mutations which lead to genetic instability $(G)$, or mutations which increase the proliferation rate $(R)$. These mutations are acquired at rate $k_{1}$. After a mutation in $G$, the mutation rate increases to $k_{2}$. Cells with one mutation go on to acquire two, three, and four mutations, denoted by listing the mutations together in alphabetical order for the cases of two and three mutations. When a cell has acquired all four mutations, it becomes a primary tumor cell $(T)$. Finally, tumor cells become metastatic cells $(M)$ at rate $k_{3}$. Double and triple state transitions are also allowed, as detailed in the text, but are not shown in this diagram for simplification. Cell birth rates $(1 / b)$ and cell death rates $(1 / d)$ have units days $^{-1}$. 
Table 1. Parameters appearing in the ODE model.

\begin{tabular}{|c|c|c|c|}
\hline Characteristic & Parameter & Value & Reference \\
\hline \hline Mutation rate without a $G$ mutation & $k_{1}$ & $10^{-7}$ mut./gene/cell div. & (Jackson and Loeb, 1998) \\
\hline Mutation rate with a $G$ mutation & $k_{2}$ & $10^{-4}$ mut./gene/cell div. & (Tomlinson et al., 1996) \\
\hline Metastasis rate & $k_{3}$ & $10^{-9} /$ cell division & (Luebeck and Moolgavkar, 2002) \\
\hline Cell division rate without an $R$ mutation & $1 / b$ & $1 / 10.00$ days $^{-1}$ & (Rew and Wilson, 2000) \\
\hline Cell division rate with an $R$ mutation & $1 / b_{R}$ & $1 / 9.89$ days $^{-1}$ & see section 4 \\
\hline Cell death rate without a $D$ mutation & $1 / d$ & $1 / 10.00$ days $^{-1}$ & (Rew and Wilson, 2000) $^{\text {see section } 4}$ \\
\hline Cell death rate with a $D$ mutation & $1 / d_{D}$ & $1 / 10.16$ days $^{-1}$ & 10 \\
\hline Cap when $\leq 10 \%$ of cells have $A$ & & $10^{6}$ cells & (Folkman, 1990) \\
\hline Lethal tumor burden cap on non- $N$, non- $M$ cells & & $10^{13}$ cells & (Friberg and Mattson, 1997) \\
\hline
\end{tabular}

In the next section, we present an ODE model that will be used to explore the following areas:

1. The kinetics of various paths to cancer.

2. The effect of inherited mutations on cancer development.

3. A sensitivity analysis of variations in the parameters.

\section{Construction of the ODE model}

Based on the basic rules outlined in the state diagram in Figure we construct 17 ODEs to model a heterogeneous population of cells undergoing the multistep process of tumorigenesis. Each equation represents one of the 17 populations of cells depicted in the state diagram and has the following format: the population of cells in a state is increased by cells gaining mutations and entering that state from previous states, is increased by cells replicating and remaining in that state, and is decreased by cells gaining new mutations and leaving that state for a new state. The populations are capped by two logistic terms, as detailed below.

We condense the ODEs into vector format as follows:

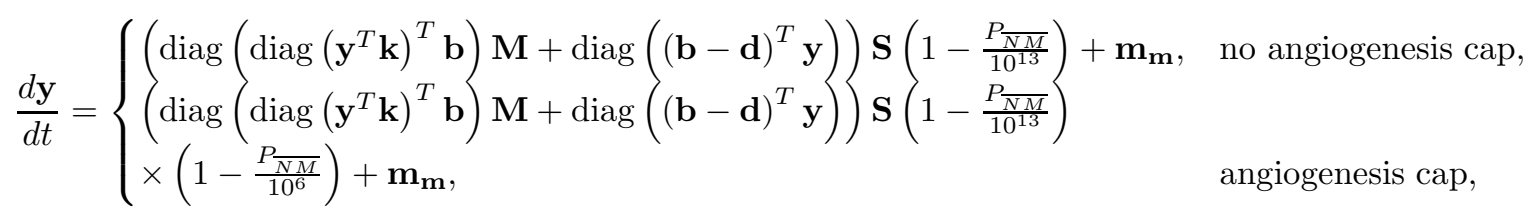

where $\mathbf{y}$ is the row vector of cell populations; $y_{1}$ is the population of normal cells, $y_{2}, y_{3}, \ldots, y_{15}$ are the populations of cells with single, double, and triple mutations, $y_{16}$ is the number of primary tumor cells (cells with all four mutations), and $y_{17}$ is the number of metastatic cells. Here, diag $(\cdot)$ is the operator which forms the row vector of the main diagonal of the matrix. The corresponding rate (row) vector is $\mathbf{k}$, with mutation rates $k_{i}$ (mutations/gene/cell division) corresponding to the mutation rate for element $y_{i}$ in $\mathbf{y}$. The same applies to the birth rates $\mathbf{b}\left(\right.$ day $\left.^{-1}\right)$ and death rates $\mathbf{d}\left(\right.$ day $\left.^{-1}\right)$. The metastasis rate vector is $\mathbf{m}_{\mathbf{m}}=\left(0,0, \ldots, 0,10^{-9} \times y_{16}\right)+$ $\left(1 / b_{R}-1 / d_{D}\right) y_{17}$, corresponding to cells leaving $y_{16}$ for $y_{17}$ at rate $10^{-9}$, and a doubling of metastatic cells at rate $\left(1 / b_{R}-1 / d_{D}\right)$ for $1 / b_{R}$ and $1 / d_{D}$ as given in Table 1 The $17 \times 17$ upper triangular matrix $\mathbf{M}$ consists of elements $M_{i, j}(j \neq i)$ for the number of genes associated with going from state $i$ to state $j$, and

$$
M_{i, i}=-\sum_{j \neq i} M_{i, j}
$$


is the main diagonal containing the number of genes for leaving each of the states. $\mathbf{S}$ is the $17 \times 17 \mathrm{matrix}$

$$
\mathbf{S}=\left(\begin{array}{ccccc}
0 & 0 & 0 & \ldots & 0 \\
0 & 1 & 0 & \ldots & 0 \\
\vdots & & \ddots & & \vdots \\
0 & \ldots & & 1 & 0 \\
0 & \ldots & & 0 & 0
\end{array}\right)
$$

used to apply the cell population caps to the non-normal, non-metastatic cells. Non-normal, non-metastatic cells are denoted by $P_{\overline{N M}}$, where

$$
P_{\overline{N M}}=\left(\sum_{i=2}^{16} y_{i}\right)
$$

The system is capped at $10^{6}$ cells using a logistic term if $<10 \%$ of the non-normal, non-metastatic cells are in states with angiogenesis mutations, otherwise this term is removed. The populations of non-normal, nonmetastatic cells are also capped by a lethal tumor burden limit of $10^{13}$ cells (Friberg and Mattson, 1997), irrespective of the angiogenesis cap. The ODEs are solved using the Runge-Kutta method of order 5, with a variable step size between 1 and $10^{-5}$, to guarantee the errors in calculating the populations remain within $10^{-4}$.

Below, we have reproduced the normal cell and four single state ODEs from the compact vector form for ease of comprehension. Note that our equations assume a constant, renewing population of normal cells, since we assume that cells leaving state $N$ for other states are few enough in number so as not to affect the population of $N$ cells.

$$
\begin{aligned}
\frac{d P_{N}}{d t} & =0 \\
\frac{d P_{A}}{d t} & =\left(\frac{100 P_{N} k_{1}}{b}-\frac{(3 \times 100+3 \times 10+1) P_{A} k_{1}}{b}\right)\left(1-\frac{P_{\overline{N M}}}{10^{6}}\right)\left(1-\frac{P_{\overline{N M}}}{10^{13}}\right), \\
\frac{d P_{D}}{d t} & =\left(\frac{100 P_{N} k_{1}}{b}+P_{D}\left(\frac{1}{b}-\frac{1}{d_{D}}\right)-\frac{(3 \times 100+3 \times 10+1) P_{D} k_{1}}{b}\right)\left(1-\frac{P_{\overline{N M}}}{10^{6}}\right)\left(1-\frac{P_{\overline{N M}}}{10^{13}}\right), \\
\frac{d P_{G}}{d t} & =\left(\frac{100 P_{N} k_{1}}{b}-\frac{(3 \times 100+3 \times 10+1) P_{G} k_{2}}{b}\right)\left(1-\frac{P_{\overline{N M}}}{10^{6}}\right)\left(1-\frac{P_{\overline{N M}}}{10^{13}}\right), \\
\frac{d P_{R}}{d t} & =\left(\frac{100 P_{N} k_{1}}{b}+P_{R}\left(\frac{1}{b_{R}}-\frac{1}{d}\right)-\frac{(3 \times 100+3 \times 10+1) P_{R} k_{1}}{b_{R}}\right)\left(1-\frac{P_{\overline{N M}}}{10^{6}}\right)\left(1-\frac{P_{\overline{N M}}}{10^{13}}\right),
\end{aligned}
$$

The equations for the other populations follow the same format and can be derived from the state diagram and from the vector form of the ODEs. In words, Equation $5 \mathrm{c}$ for example, says that the population of cells with a mutation in $D$ is increased by normal cells gaining a mutation in one of 100 genes in $D$ at a rate of $k_{1}$ every $b$ days. The population is also increased by cells in state $D$ replicating (but not mutating) every $b$ days and dying every $d_{D}$ days. The population is decreased by cells leaving state $D$ and gaining a single mutation in one of 3 other categories $(A D, D G, D R$ ) each with 100 genes, by gaining a double mutation in one of 3 ways ( $D G R$, $A D G, A D R$ ), with 10 genes being involved in each transition, or by gaining a triple mutation to go to state $A D G R$ with 1 gene being involved in the transition. The logistic term caps the total population of non-normal, non-metastatic cells at $10^{6}$ cells. What is not visible in this standard form of the ODEs but is present in the vector form is the fact that the logistic angiogenesis cap is only imposed when less than 10\% of the non-normal, non-metastatic cells have a mutation in the $A$ category. Finally, populations of non-normal, non-metastatic cells are always capped by a lethal tumor burden limit of $10^{13}$ cells. 


\section{Calculation of cell division and cell death rates}

In order to calculate the change in cell division and cell death rates for mutations in $R$ and $D$, we begin with the assumption that birth and death rates are equal for normal cells, i.e. cell division rate $=1 / b=$ cell death rate $=1 / d=1 / 10$ days $^{-1}$.

We use an approximation to the ODEs where we treat the rate of cells entering and leaving the state as negligible compared with the tumor volume doubling time, since they are several orders of magnitude different. Thus, for cells with mutations in $D$ but not $R$, say, we consider

$$
\frac{d D}{d t}=D\left(\frac{1}{b}-\frac{1}{d_{D}}\right) .
$$

Solving this gives $D=D_{0} \exp \left(\left(1 / b-1 / d_{D}\right) t\right)$. A doubling corresponds to $2=\exp \left(\left(1 / b-1 / d_{D}\right) T_{D}\right)$. Similarly, for cells with mutations in $R$ and not $D$, we arrive at $R=R_{0} \exp \left(\left(1 / b_{R}-1 / d\right) t\right)$. Taking the natural logarithm of both sides leads to Equations 7 and 8

$$
\begin{aligned}
& \frac{\ln 2}{1 / b_{R}-1 / d}=T_{R}, \\
& \frac{\ln 2}{1 / b-1 / d_{D}}=T_{D},
\end{aligned}
$$

where $T_{R}$ is tumor volume doubling time for cells with a mutation in $R$ but not $D$ and equals $T+50 \times 3$, where $T_{D}$ is the tumor volume doubling time for cells with a mutation in $D$ but not $R$ and equals $T+50 \times 7$, and where the $D: R$ importance ratio is $0.7: 0.3$. The base tumor volume doubling time is $T$ when the growing tumor has mutations in both $D$ and $R$ (500 days). The value 50 is chosen to give realistic doubling times for cells with mutations in $D$ (but not $R$ ) and $R$ (but not $D$ ), on the upper bound of observed tumor volume doubling times, where the cells typically have both mutations.

\section{$5 \quad$ Kinetics of various paths to cancer}

Given that multiple mutations are necessary to form a tumor, we are interested in whether the specific order of mutations is important. It is currently believed that the temporal sequence of mutations determines the propensity of tumor development (Arends, 2000). The extent to which genetic instability $(G)$ determines the timing of tumorigenesis has been a controversial issue in cancer biology. Some have argued that an increased premalignant mutation rate (that is, acquiring a mutation in $G$ early) is necessary for tumor development (Loeb, 1991: Raiagopalan et al., 2003). Others have argued that an increased cell division rate, offering more opportunities to accumulate mutations, is sufficient for tumorigenesis (Tomlinson and Bodmer, 1995, 1999; Sieber et al., 2003). Although the extent to which angiogenesis $(A)$, decreased apoptosis $(D)$, genetic instability $(G)$, and increased replication rate $(R)$ contribute to the development of cancer depends on the type of cancer involved, a better general understanding of the kinetics of various paths to cancer can be informative about their relative importance.

We explore the kinetics of various pathways to cancer by analyzing the dynamics of the different cell populations. By plotting different sets of cell populations, we are able to identify the individual contribution of each mutation to the development of cancer. The growing populations of cells plateau at various points in the graphs due to the imposed $10^{13}$ cell population cap. In this model, the fastest pathway for tumor progression starts with a mutation in $D$ (Figure 2(a) which increases the population of potential tumor cells. Next, a mutation in $R$ is acquired, further increasing the population of cells by clonal expansion (Figure 2(b)). After acquiring these two mutations, the tumor is sufficiently large to be inhibited by the angiogenesis cap imposed by the model. For this reason, a mutation in the angiogenesis category occurs next in the fastest path (Figure 2(c)]. Finally, a mutation in $G$ follows. Figure 2(d) shows the populations of tumor cells, $T$, and metastatic cells, $M$. Although 
the rate $k_{3}=10^{-9}$ is very low, the large increase in population of $T$ cells guarantees that eventually some cells successfully metastasize.

Our model predicts that genetic instability is more likely to be a feature of later-stage sporadic tumors, in accordance with the view of Tomlinson and Bodmer (1999). This is because a mutation in $G$ has no direct selective advantage, only an indirect advantage through increasing the mutation rates in other genes. Although genetic instability can aid tumorigenesis, selection and clonal expansion are the main driving force for tumor progression in this model, a conclusion which has been proposed previously by Sieber et al. (2003).

\section{Effect of inherited mutations on cancer development}

Here we examine the effect of different inherited mutations on cancer development by varying our initial conditions. Since most inherited cancers are the result of mutations in tumor suppressors (Knudson, 2002), we model this situation by increasing the rate of transition from a normal cell to the appropriate mutated cell to $10^{-5}$ mutations/gene/cell division. This models a case where a person inherits an inactivating mutation in one allele of the gene. These cells are still functionally "normal" (thus they begin in state $N$ ), but the chance of acquiring the second "hit" and losing functionality of the protein (moving into the mutated state) is much increased.

As expected, inheriting a mutation in a cancer-critical gene decreases the time to cancer onset. The effects of inheriting a mutation in each category on time to reach $10^{9}$ primary tumor cells, $10^{12}$ primary tumor cells, and $10^{12}$ metastatic cells are shown in Figure 3 A tumor volume of 1 cubic centimeter weighs about 1 gram and represents about $10^{9}$ cells (Friberg and Mattson, 1997). This tumor size is regarded as relatively small in a clinical setting and it is at this size that a tumor may give rise to the first symptoms and may first become detectable by palpation (Friberg and Mattson, 1997). A tumor that weighs about 1 kilogram ( $10^{12}$ cells) is approaching the lethal tumor burden for a patient (Friberg and Mattson, 1997). The $10^{12}$ metastatic cells plotted in Figure 3 are not necessarily localized to one site in the body; they could represent $10^{12}$ cells present in one location or $10^{11}$ cells present in each of 10 different locations, for example.

In contrast to the results obtained in 4.1 .1 where the increased population of cells caused by mutations in $D$ and $R$ dominates the fastest path to sporadic cancer, inheriting a mutation in a $G$ gene causes cancer onset at the earliest age. There is no observable difference between inheriting a mutation in one of the other categories and inheriting no mutations at all. In the fastest path plots (Figure 2), there is equal probability of acquiring a mutation in $A, D, G$ or $R$. $D$ will dominate over $G$ due to the fact that the transition from one state to another is a function not only of the mutation rates $k_{1}$ and $k_{2}$ but also the cell population size. Both $D$ and $G$ are equally likely to begin with, but since $D$ increases the net cell population very quickly, it soon dominates over the rate $k_{2}$ associated with $G$. Therefore, the fastest path to sporadic cancer is through a mutation in $D$ first.

In comparison, when a mutation in $G$ is inherited, the cell has already surpassed the initial probability hurdle of acquiring a mutation in $G$. The rate of subsequent mutation is now 1000-fold higher and once a mutation in $D$ or $R$ is obtained, the cell population will begin to increase. For this reason, an inherited mutation in $G$ has the greatest effect. This is consistent with the fact that many inherited cancer syndromes are the result of a mutation in the $G$ category. These include xeroderma pigmentosum, ataxia telangiectasia, Nijmegen breakage syndrome, hereditary non-polyposis colorectal cancer, and Bloom syndrome (Sieber et al., 2003).

The time to develop a palpable primary tumor $\left(10^{9}\right.$ cells $)$ in this model is 16.25 years if no mutations are inherited (Figure 31). Even taking into account the fact that detection of the tumor would not occur until several years later (Friberg and Mattson, 1997), this age of cancer onset is significantly earlier than the average age of cancer onset in the human population (DePinho, 2000). This is an indication of the need for more accurate information on cell division, cell death, and tumor doubling rates. Importantly, this may also be an indication that acquisition of mutations in more than four categories is necessary for development of a primary tumor. Adding two more steps to the multistep model would certainly delay the time to cancer, and would be more in accordance with the six-step model proposed by Hanahan and Weinberg (2000). Consideration of the role of the immune system 


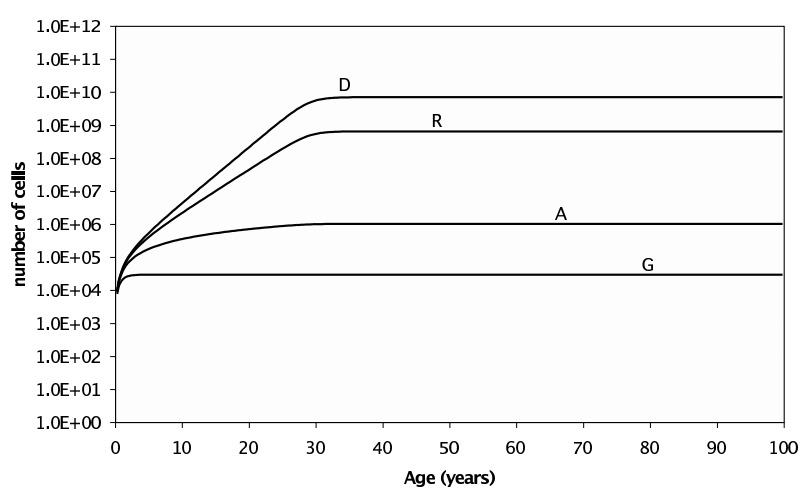

(a)

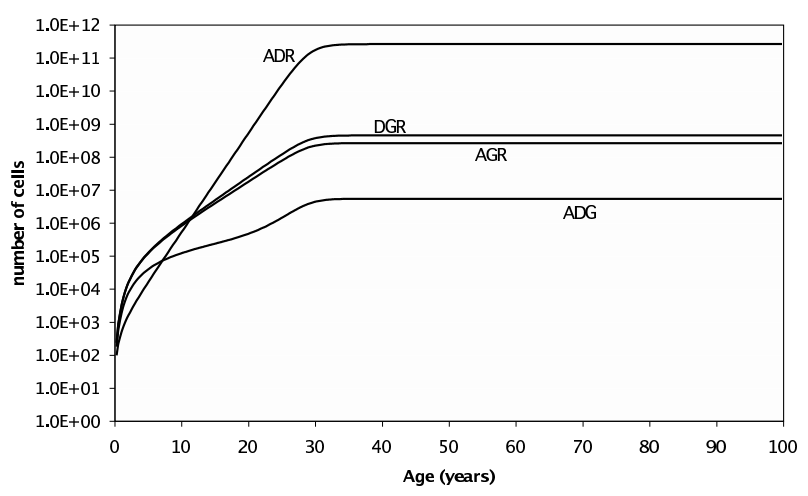

(c)

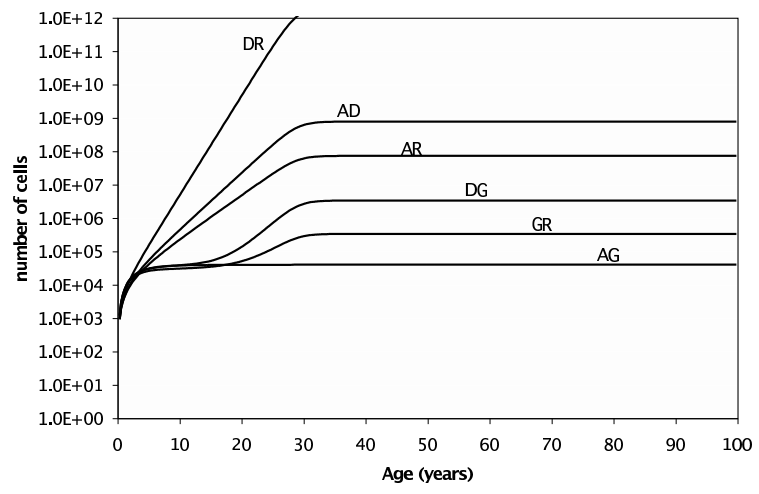

(b)

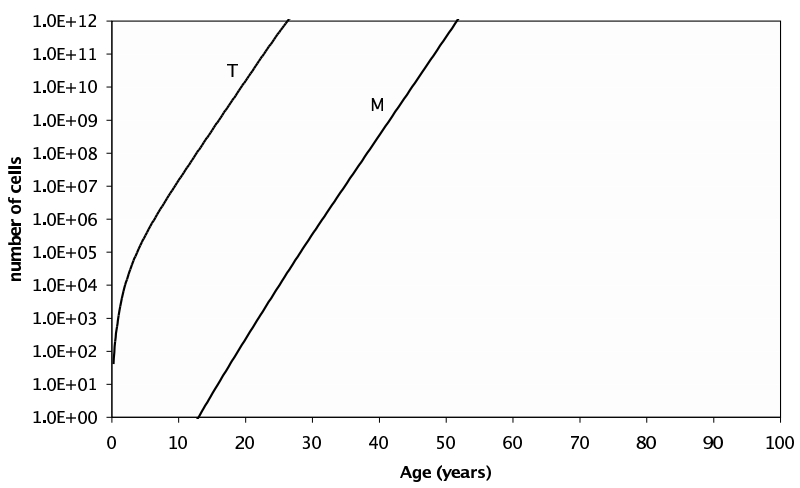

(d)

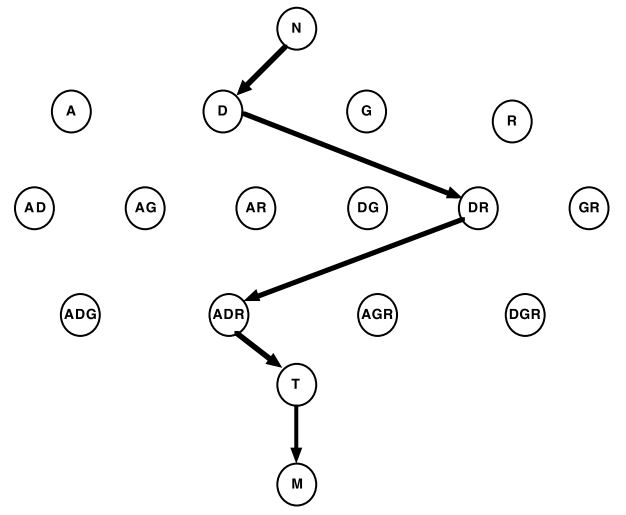

(e)

Figure 2. Fastest path to cancer. (a) Dynamics of cell populations with one type of mutation. (b) Dynamics of cell populations with two types of mutations. (c) Dynamics of cell populations with three types of mutations. (d) Dynamics of cell populations with four types of mutations $(T)$, and those that have metastasized $(M)$. (e) The fastest path to cancer is by acquiring a mutation in $D$, then $R$, then $A$, then $G$.

in curbing the growth of a tumor would also slow the time to cancer onset. Our model does not directly consider this factor, although category $D$ does allow for apoptosis initiated by the immune system. Consideration of these three factors would allow the model to be more appropriately scaled to the timing of human cancer. 


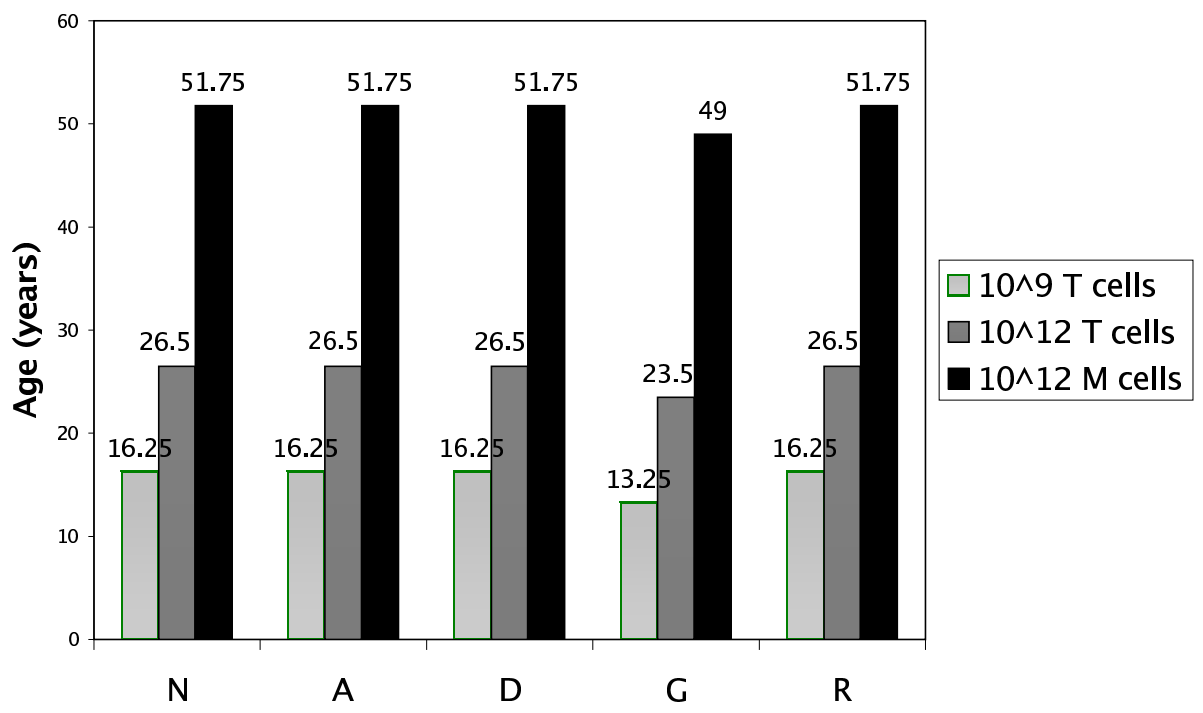

Figure 3. Inherited mutations in cancer-critical genes. Age at which a person may acquire $10^{9}$ primary tumor cells, $10^{12}$ primary tumor cells, and $10^{12}$ metastatic cells with different inherited mutations. For reference, the case where no mutations are inherited is also shown $(N)$.

\section{Sensitivity analysis of variations in the parameters}

In order to determine the relative contributions of the parameters to the model, we vary each parameter listed in Table 2 while holding all others constant at the default value. The default values chosen are our best estimate from the literature. Except for $D: R$ importance ratio where we use 0.3:0.7 to determine the effect on the fastest path, and $\%$ A cells needed to remove cap where we test the range from $0 \%$ to $100 \%$, the other values are chosen to be near the upper and lower bounds of the range given in the literature. We assess the contribution of each by examining their effect on time to reach $10^{12} M$ cells and on the fastest pathway to cancer in Table 2

The most salient result of the sensitivity analysis is the robustness of the model. Despite trials with very high values for $k_{2}$, the fastest path to somatic cancer is always via a mutation in $D$ then $R$ then $A$ then $G$, except in the case where we flip the $D: R$ importance ratio. As expected, a ratio of 0.3:0.7 flips the roles of $D$ and $R$ in the fastest path to give $R D A G$, but does not change the time to $10^{12} M$ cells from the default value of 51.75 years. Using a $D: R$ ratio of 0.8:0.2 decreases the time to reach $10^{12} M$ cells due to the increased weight given to $D$.

The parameter that has the largest effect on time to reach $10^{12} M$ cells is the tumor volume doubling time. A tumor volume doubling time of 300 days decreases the time to reach $10^{12} M$ cells by 13.50 years relative to the default of 500 days, and a tumor volume doubling time of 700 days increases the time to reach $10^{12} M$ cells by 13.00 years. This effect is seen in Figure 4(a) as well as in Table 2 The large effect of this parameter on the model is due to its impact on the $\left(\frac{1}{b}-\frac{1}{d}\right)$ term; when cells have mutations in $R$ and/or $D$, the terms become $1 / b_{R}$ and/or $1 / d_{D}$, allowing the cell populations to increase at a rate that reflects the tumor volume doubling time chosen.

Variations in the birth and death rates to 1 every 5 days and 1 every 30 days also have an effect on time to reach $10^{12} M$ cells. This can be seen in row one of Table 2 but the effect is small when compared with the effect of tumor volume doubling time.

The time (51.75 years) to reach $10^{12} M$ cells does not change in varying the percentage of $A$ cells needed to remove the angiogenesis cap from $0 \%$ to $31 \%$. Between $31 \%$ and $35 \%$, the time to reach $10^{12} M$ cells increases rapidly. The time (57.50 years) to reach $10^{12} \mathrm{M}$ cells does not change in varying the percentage from $35 \%$ to $100 \%$. This effect can be seen in Figure 4(b) At 31\%, the requirement for mutations in the $A$ category begins to have an effect on the growing cell populations. At $35 \%$ and above, the percentage of $A$ cells required is so large 
that sum of the populations of non-normal, non-metastatic cells never gets above $10^{6}$ because there are never at least $35 \%$ with $A$ mutations. Thus the time to reach $10^{12} \mathrm{M}$ cells depends only on a fixed number of $T$ cells in each case, and remains constant at 57.50 years for percentages $35 \%$ and above.

A ten-fold change (from $10^{-7}$ to $10^{-6}$ mutations/gene/cell division) in mutation rate without a $G$ mutation $\left(k_{1}\right)$ has a larger effect on time to reach $10^{12} M$ cells than a ten-fold change (from $10^{-4}$ to $10^{-3}$ mutations/gene/cell division) in mutation rate with a $G$ mutation $\left(k_{2}\right)$. This is due to the fact that the effect of $k_{2}$ only becomes important later in tumorigenesis since $G$ is last in the fastest path to cancer, whereas the effect of $k_{1}$ occurs at the beginning. There is no change in time to reach $10^{12} \mathrm{M}$ cells when $k_{2}$ is increased from $10^{-3}$ to $10^{-2}$ mutations/gene/cell division because the effect of mutation rate has already saturated the system at a $k_{2}$ value of $10^{-3}$.

Increasing the number of genes involved in the transitions decreases time to reach $10^{12} M$ cells simply due to the larger numerator in the differential equations.

Table 2. Sensitivity of the model to changes in changes in parameters. Cell birth and death rates have units days ${ }^{-1}$. Tumor volume doubling times are measured in days. Mutation rates are measured as mutations/gene/cell division. Number of genes involved in transitions are listed as number involved in single, double, triple transitions. "Other" refers to different values tested in the sensitivity analysis. "Time" refers to age at acquisition of $10^{12} M$ cells for a variation in that parameter, measured in years. "Path" refers to the fastest path to cancer for a variation in that parameter.

\begin{tabular}{|c|c|c|c|c|c|c|c|c|c|}
\hline Parameter & Default & Time & Path & Other & Time & Path & Other & Time & Path \\
\hline Cell birth and death rates & $1 / 10$ & $\overline{51.75}$ & DRAG & $1 / 5$ & $\overline{50.25}$ & DRAG & $1 / 30$ & $\overline{54.75}$ & DRAG \\
\hline Tumor volume doubling time & 500 & 51.75 & DRAG & 300 & 38.25 & DRAG & 700 & 64.75 & DRAG \\
\hline$\% A$ cells needed to remove cap & $10 \%$ & 51.75 & DRAG & $30 \%$ & 51.75 & DRAG & $40 \%$ & 57.50 & DRAG \\
\hline$D: R$ importance ratio & $0.7: 0.3$ & 51.75 & DRAG & $0.8: 0.2$ & 50.50 & DRAG & $0.3: 0.7$ & 51.75 & RDAG \\
\hline Mut. rate with a $G$ mutation & $10^{-4}$ & 51.75 & DRAG & $10^{-3}$ & $\overline{50.50}$ & $\overline{\text { DRAG }}$ & $10^{-2}$ & $\overline{50.50}$ & $\overline{\mathrm{DRAG}}$ \\
\hline Mut. rate without a $G$ mutation & $10^{-7}$ & 51.75 & DRAG & $10^{-6}$ & 48.50 & DRAG & & & \\
\hline \# of genes involved in transitions & $\overline{100,10,1}$ & 51.75 & DRAG & $500,100,10$ & 48.25 & DRAG & & & \\
\hline
\end{tabular}

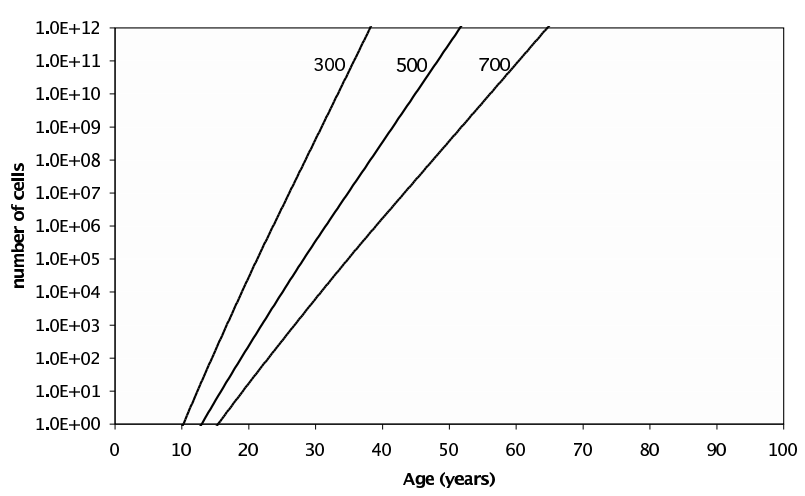

(a)

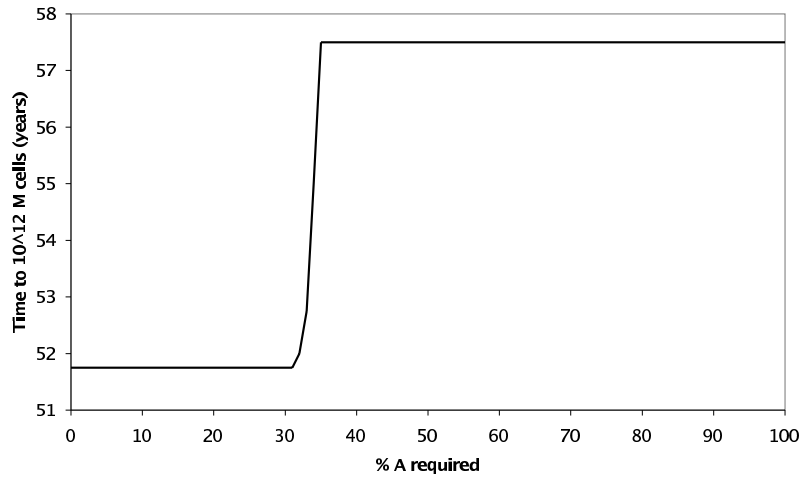

(b)

Figure 4. Sensitivity of the model to changes in parameters. (a) Sensitivity to changes in tumor volume doubling time. Time to reach $10^{12} \mathrm{M}$ cells is shown for tumor volume doubling times of 300 days, 500 days, and 700 days. (b) Effect of variations in percentage of $A$ cells required to induce angiogenesis on time to reach $10^{12} M$ cells.

\section{Discussion}

This paper explores facets of the multistep model of oncogenesis. The key findings of this paper are (1) the fastest path to somatic cancer is predicted to be through gaining mutations in $D$, then $R$, then $A$, then $G,(2)$ 
of the four categories of mutations, inheriting a mutation in $G$ is predicted to produce cancer at the earliest age, and (3) the fastest path to somatic cancer is robust to realistic changes in parameters, with the model being most affected by variations in tumor volume doubling time.

The strength of our model lies not in its utility for predicting any one individual's time to cancer onset per se, but rather in the fact that it presents a novel approach to understanding the genetic basis of cancer from a systems biology perspective. Although a thorough testing of this model is not currently possible due to lack of appropriate biological data, this model establishes the groundwork for future models that can be directly tied to clinical and molecular data. We hope that the creation of this model for the multistep progression to cancer will encourage biologists to gather quantitative data and will suggest which experiments should be performed with highest priority. The only parameter values which are reasonably agreed upon in the literature are the spontaneous mutation rate and the size to which a tumor can grow before angiogenesis is required. All other parameter values could use experimental refinement, especially tumor volume doubling time, since it has the largest effect on time to cancer onset. Better estimates of parameter values, inclusion of two additional categories to give a total of six steps in the multistep model, and consideration of the role of the immune system in curbing the growth of a tumor will allow our model to be more appropriately scaled to human cancers. Modeling the multistep accumulation of genetic mutations in cancer will give insight into topical questions about the progression of a normal cell to a cancerous cell, enabling cancer treatments to be better targeted to various stages of cancer progression, and suggesting the most important directions for future experimental research.

\section{Acknowledgements}

We thank Trachette Jackson, Gilbert Omenn, John Holland (University of Michigan), Andrew Allison, Samuel Mickan, David Findlay, Brendon Coventry (The University of Adelaide), Setayesh Behin-Ain (The Queen Elizabeth Hospital, Australia), and Roger Reddel (Children's Medical Research Institute, Australia) for useful discussions, Sara Dempster (Massachusetts Institute of Technology) for her early contributions to this project, and the Santa Fe Institute for the opportunity to attend the Complex Systems Summer School where we began our exploration of this topic. This work was supported in part by scholarship funding from The Queen Elizabeth Hospital, Adelaide, Australia, arranged through Setayesh Behin-Ain.

\section{References}

Amatschek, S., Koenig, U., Auer, H., Steinlein, P., Pacher, M., Gruenfelder, A., Dekan, G., Vogl, S., Kubista, E., Heider, K., Stratowa, C., Schreiber, M., Sommergruber, W., 2004. Tissue-wide expression profiling using cdna subtraction and microarrays to identify tumor-specific genes. Cancer Research 64, 844-856.

Arends, J., 2000. Molecular interactions in the Vogelstein model of colorectal carcinoma. Journal of Pathology $190,412-416$.

Armitage, P., Doll, R., 1954. The age distribution of cancer and a multi-stage theory of carcinogenesis. British Journal of Cancer 8, 1-12.

Cahill, D., Kinzler, K., Vogelstein, B., Lengauer, C., 1999. Genetic instability and darwinian selection in tumours. Trends Cell Biology 9, M57-M60.

DePinho, R., 2000. The age of cancer. Nature 408, 248-254.

Ernst, T., Hergenhahn, M., Kenzelmann, M., Cohen, C., Bonrouhi, M., Weninger, A., Klaren, R., Grone, E., Wiesel, M., Gudemann, C., Kuster, J., Schott, W., Staehler, G., Kretzler, M., Hollstein, M., Grone, H., 2002. Decrease and gain of gene expression are equally discriminatory markers for prostate carcinoma. American Journal of Pathology 160, 2169-2180. 
Folkman, J., 1990. What is the evidence that tumors are angiogenesis dependent? Journal of the National Cancer Institute $82,4-6$.

Friberg, S., Mattson, S., 1997. On the growth rates of human malignant tumors: Implications for medical decision making. Journal of Surgical Oncology 65, 284-297.

Hanahan, D., Weinberg, R., 2000. The hallmarks of cancer. Cell 100, 57-70.

Jackson, A., Loeb, L., 1998. The mutation rate and cancer. Genetics 148, 1483-1490.

Knudson, A., 2002. Cancer genetics. American Journal of Medical Genetics 111, 96-102.

Loeb, L., 1991. Mutator phenotype may be required for multistage carcinogenesis. Cancer Research 51, 30753079 .

Luebeck, E., Moolgavkar, S., 2002. Multistage carcinogenesis and the incidence of colorectal cancer. PNAS 99, 15095-15100.

Nakamura, T., Furukawa, Y., Nakagawa, H., Tsunoda, T., Ohigashi, H., Murata, K., Ishikawa, O., Ohgaki, K., Kashimura, N., Miyamoto, M., Hirano, S., Kondo, S., Katoh, H., Nakamura, Y., Katagiri, T., 2004. Genomewide cDNA microarray analysis of gene expression profiles in pancreatic cancers using populations of tumor cells and normal ductal epithelial cells selected for purity by laser microdissection. Oncogene 23, Epub.

Nowell, P., 1976. The clonal evolution of tumor cell populations. Science 194, 23-28.

Rajagopalan, H., Nowak, M., Vogelstein, B., Lengauer, C., 2003. The significance of unstable chromosomes in colorectal cancer. Nature Reviews Cancer 3, 695-701.

Renan, M., 1993. How many mutations are required for tumorigenesis? implications from human cancer data. Molecular Carcinogenesis 7, 139-146.

Rew, D., Wilson, G., 2000. Cell production rates in human tissues and tumours and their significance. Part II: clinical data. European Journal of Surgical Oncology 26, 405-417.

Sieber, O., Heinimann, K., Tomlinson, I., 2003. Genomic instability - the engine of tumorigenesis? Nature Reviews Cancer 3, 701-708.

Tomlinson, I., Bodmer, W., 1995. Failure of programmed cell death and differentiation as causes of tumors some mathematical models. PNAS 92, 11130-11134.

Tomlinson, I., Bodmer, W., 1999. Selection, the mutation rate and cancer: ensuring that the tail does not wag the dog. Nature Medicine 5, 11-12.

Tomlinson, I., Novelli, M., Bodmer, W., 1996. The mutation rate and cancer. PNAS 93, 14800-14803.

Vogelstein, B., Lane, D., Levine, A., 2000. Surfing the p53 network. Nature 408, 307-310.

Zou, T., Selaru, F., Xu, Y., Shustova, V., Yin, J., Mori, Y., Shibata, D., Sato, F., Wang, S., Olaru, A., Deacu, E., Liu, T., Abraham, J., Meltzer, S., 2002. Application of cDNA microarrays to generate a molecular taxonomy capable of distinguishing between colon cancer and normal colon. Oncogene 21, 4855-4862. 